\title{
LOCAL AND GLOBAL STRONG SOLUTION BY THE SEMI-GALERKIN METHOD FOR THE MODEL OF MASS DIFFUSION
}

\author{
P. D. Damázio \\ F. Guillén-González \\ J. V. Gutiérrez-Santacreu M. A. Rojas-Medar
}

\begin{abstract}
In this work, we present some results for local and global in time solutions (defined in the time interval $(0, T)$ with $T<+\infty$ or $T=$ $+\infty$ ) for the model of mass diffusion by using the spectral semi-Galerkin approximations. We establish results related to the global existence of weak solutions, to the existence of strong solutions (local in time or global in time for small enough data in $3 D$ domains or general data in $2 D$ case), to the regularity of strong solutions and the effects of the exponential decay of the external force in the asymptotic behavior when $t \rightarrow+\infty$ for global solutions.
\end{abstract}

\section{Introduction}

\section{$1.1 \quad$ Model}

Let $\Omega \subseteq \mathbb{R}^{d}(d=2$ or 3$)$ be a bounded domain with boundary $\Gamma$ of class $C^{1,1}$. We will use the notation $Q=\Omega \times(0, T), \Sigma=\Gamma \times(0, T)$ being $0<T \leq+\infty$ and $\boldsymbol{n}$ the unit outwards normal vector on $\Gamma$. We consider the following initial-value problem concerning fluids with mass diffusion

$$
\left\{\begin{array}{l}
\rho \boldsymbol{u}_{t}+((\rho \boldsymbol{u}-\lambda \nabla \rho) \cdot \nabla) \boldsymbol{u}-\mu \Delta \boldsymbol{u}-\lambda(\boldsymbol{u} \cdot \nabla) \nabla \rho+\nabla p \\
+\lambda^{2}\left(\nabla \cdot\left(\frac{1}{\rho} \nabla \rho \otimes \nabla \rho\right)\right)=\rho \boldsymbol{f} \text { in } Q, \\
\nabla \cdot \boldsymbol{u}=0 \text { in } Q,\left.\quad \boldsymbol{u}\right|_{\Sigma}=0, \quad \boldsymbol{u}(0)=\boldsymbol{u}_{0} \quad \text { in } \Omega, \\
\rho_{t}-\lambda \Delta \rho+\boldsymbol{u} \cdot \nabla \rho=0 \text { in } Q,\left.\quad \frac{\partial \rho}{\partial \boldsymbol{n}}\right|_{\Sigma}=0, \quad \rho(0)=\rho_{0} \quad \text { in } \Omega,
\end{array}\right.
$$

where the unknows are $\boldsymbol{u}$ the incompressible velocity, $p$ the pressure and $\rho$ the fluid density. The data are, $\boldsymbol{f}$ the external force acting on the system and 
$\mu>0, \lambda>0$ the viscosity and density coefficients respectively.

An extensive physical discussions and derivation of problem (1) can be seen in Frank-Kamenestskii [5], Kazhikhov and Smagulov [12], Antoncev, Kazhikhov and Monakhov [1].

Throughout this paper we will assume that there exists some constants $m, M>0$, such that

$$
0<m \leq \rho_{0} \leq M \quad \text { in } \Omega .
$$

\subsection{Known results}

For the model (1) considered in this paper, Beirão da Veiga [2] and Secchi [17], established the local existence of strong solutions by using linearization and fixed point argument. Indeed, in [2] the local existence of strong solutions (defined in $(0, T)$ ) imposing smallness constraints on data or on final time for $3 D$ domains is proved. In [17], $\lambda / \mu$ small enough is imposed in the $2 D$ case, obtaining existence and uniqueness of global strong solution (defined in $(0, T))$. Moreover, it is showed the convergence, as $\lambda \rightarrow 0$, towards a weak solution of the Navier-Stokes problem with variable density. In the $3 D$ case, global existence of $(1)$ and convergence (as $\lambda \rightarrow 0$ ) towards weak solutions of Navier-Stokes with variable density is proven in [7], imposing only positive initial density $\left(\rho_{0} \geq 0\right)$. In [9], the existence and regularity of strong solutions (and some error estimates) is proved by means of an iterative method.

The case where the solution is defined in $t \in(0,+\infty)$ is treated in [3], imposing that the external force $\mathbf{f} \in L^{\infty}\left(0,+\infty ; L^{2}(\Omega)\right)$ and periodic in time and studies the asymptotic behavior of the solution as $t \rightarrow+\infty$. 


\subsection{Some functional spaces and semi-Galerkin method}

In the sequel, $(\cdot, \cdot)$ denotes the $L^{2}$ inner product. Also, $\|\cdot\|$ will denote the $L^{2}$ norm. We now introduce the standard spaces of the Navier-Stokes framework:

$$
\begin{aligned}
& \boldsymbol{H}=\left\{\boldsymbol{u}: \boldsymbol{u} \in \boldsymbol{L}^{2}(\Omega), \nabla \cdot \boldsymbol{u}=0, \boldsymbol{u} \cdot \boldsymbol{n}=0 \text { on } \partial \Omega\right\}, \\
& \mathbf{V}=\left\{\boldsymbol{u}: \boldsymbol{u} \in \boldsymbol{H}^{1}(\Omega), \operatorname{div} \mathbf{u}=0, \boldsymbol{u}=0 \text { on } \partial \Omega\right\}, \\
& L_{0}^{2}(\Omega)=\left\{p: p \in L^{2}(\Omega), \int_{\Omega} p(\mathbf{x})=0\right\} .
\end{aligned}
$$

The norms $\|\boldsymbol{u}\|_{H^{1}}$ and $\|\nabla \boldsymbol{u}\|_{L^{2}}$ are equivalent in $\boldsymbol{V}$, and $\|\boldsymbol{u}\|_{H^{2}}$ and $\|A \boldsymbol{u}\|_{L^{2}}$ are equivalent in $\boldsymbol{H}^{2}(\Omega) \cap \boldsymbol{V}([13,19])$. On the other hand, the norms $\|p\|_{H^{1}}$ and $\|\nabla p\|_{L^{2}}$ are equivalent in $H^{1}(\Omega) \cap L_{0}^{2}(\Omega)$.

On the other hand, for the density, let us consider the affine space $(k=2,3)$

$$
H_{N}^{k}(\Omega)=\left\{\rho \in H^{k}(\Omega): \frac{\partial \rho}{\partial \mathbf{n}}=0 \text { on } \partial \Omega, \int_{\Omega} \rho(\mathbf{x})=\int_{\Omega} \rho_{0}(\mathbf{x})\right\} .
$$

Obviously, $H_{N}^{k}(\Omega)=\overline{\rho_{0}}+H_{N, 0}^{k}(\Omega)$, where $\overline{\rho_{0}}=\frac{1}{|\Omega|} \int_{\Omega} \rho_{0}(\mathbf{x}) d \mathbf{x}$ and

$$
H_{N, 0}^{k}(\Omega)=\left\{\rho \in H^{k}(\Omega): \frac{\partial \rho}{\partial \mathbf{n}}=0 \text { on } \partial \Omega, \int_{\Omega} \rho(\mathbf{x})=0\right\} .
$$

Therefore, $H_{N, 0}^{k}(\Omega)(k=2$ or $k=3)$ is a closed subspace of $H_{N}^{k}(\Omega)$. Consequently, thanks to the $H^{2}$ and $H^{3}$ regularity of the Poisson-Neumann problem, norms $\|\rho\|_{H^{2}}$ and $\|\Delta \rho\|_{L^{2}}$ are equivalent in $H_{N}^{2}(\Omega)$ and $\|\rho\|_{H^{3}}$ and $\|\nabla \Delta \rho\|_{L^{2}}$ are equivalent in $H_{N}^{3}(\Omega)([2])$.

We also consider the Stokes operator $A: D(A) \rightarrow \boldsymbol{H}$ defined by $A=P(-\Delta)$ with domain $D(A)=\boldsymbol{H}^{2}(\Omega) \cap \boldsymbol{V}$ where $P: \boldsymbol{L}^{2}(\Omega) \rightarrow \boldsymbol{H}$ is the Helmholtz orthogonal projection and define $\boldsymbol{V}_{k}=\left\langle\boldsymbol{u}_{1}, \ldots, \boldsymbol{u}_{k}\right\rangle$ the finite vectorial space spanned by the first $k \in \mathbb{N}$ eigenfunctions associated to the Stokes operator.

The spectral semi-Galerkin approximations of problem (1) are defined for each $k \in \mathbb{N}$ as the solution $\left(\boldsymbol{u}^{k}, \rho^{k}\right) \in C^{2}\left([0, T], \boldsymbol{V}_{k}\right) \times C^{2}\left([0, T] ; H_{N}^{3}(\Omega)\right)$ of 


$$
\left\{\begin{array}{l}
\left(\rho^{k} \boldsymbol{u}_{t}^{k}, \boldsymbol{v}\right)+\left(\left(\left(\rho^{k} \boldsymbol{u}^{k}-\lambda \nabla \rho^{k}\right) \cdot \nabla\right) \boldsymbol{u}^{k}, \boldsymbol{v}\right)+\mu\left(A \boldsymbol{u}^{k}, \boldsymbol{v}\right) \\
-\lambda\left(\left(\boldsymbol{u}^{k} \cdot \nabla\right) \nabla \rho^{k}, \boldsymbol{v}\right)+\lambda^{2}\left(\nabla \cdot\left(\frac{1}{\rho^{k}} \nabla \rho^{k} \otimes \nabla \rho^{k}\right), \boldsymbol{v}\right) \\
=\left(\rho^{k} \boldsymbol{f}, \boldsymbol{v}\right), \quad \forall \boldsymbol{v} \in \boldsymbol{V}_{k}, \text { in }(0, T) .
\end{array}\right.
$$

$$
\begin{cases}\frac{\partial \rho^{k}}{\partial t}+\boldsymbol{u}^{k} \cdot \nabla \rho^{k}-\lambda \Delta \rho^{k}=0 & \text { in } Q \\ \frac{\partial \rho^{k}}{\partial \boldsymbol{n}}=0, & \text { on } \Sigma\end{cases}
$$

with the initial conditions

$$
\boldsymbol{u}^{k}(\boldsymbol{x}, 0)=P_{k} \mathbf{u}_{0} \text { and } \rho^{k}(0, \boldsymbol{x})=\rho_{0}(\boldsymbol{x}), \quad \forall \boldsymbol{x} \in \Omega \text {. }
$$

respectively, where $P_{k}$ is the proyection operator on $\boldsymbol{V}_{k}$.

Definition 1 A pair $(\rho, \boldsymbol{u})$ is called a weak solution of $(1)$ in $\left(0, T^{\prime}\right)$ with $T^{\prime}=$ $T<\infty$ or $T^{\prime}=+\infty$ if it verifies:

a)

$$
\begin{aligned}
& (\boldsymbol{u}, \rho) \in L^{\infty}\left(0, T^{\prime} ; \boldsymbol{H} \times H_{N}^{1}(\Omega)\right), \\
& (\boldsymbol{u}, \rho) \in L^{2}\left(0, T^{\prime} ; \boldsymbol{V} \times H_{N}^{2}(\Omega)\right) \text { if } T^{\prime}<\infty \text { or }(\boldsymbol{u}, \rho) \in L_{\text {loc }}^{2}([0, \infty) ; \\
& \left.\quad \boldsymbol{V} \times H_{N}^{2}(\Omega)\right) \text { if } T^{\prime}=\infty, \\
& 0<m \leq \rho(\boldsymbol{x}, t) \leq M, \quad \text { a.e. }(\boldsymbol{x}, t) \in\left(0, T^{\prime}\right) \times \Omega .
\end{aligned}
$$

b) $\forall \phi \in C^{1}([0, t] ; \boldsymbol{V})$ such that $\phi(t)=0$, where $t \in\left(0, T^{\prime}\right)$

$$
\begin{aligned}
& \int_{0}^{t}\left\{-\left(\boldsymbol{u}, \rho \phi_{t}+(\rho \boldsymbol{u}-\lambda \nabla \rho) \cdot \nabla \phi\right)+\left(\mu \nabla \boldsymbol{u}-\lambda \rho(\nabla \boldsymbol{u})^{t}, \nabla \phi\right)\right\} d s \\
& =\int_{0}^{t}(\rho \boldsymbol{f}, \phi) d s+\left(\rho_{0} \boldsymbol{u}_{0}, \phi(0)\right) .
\end{aligned}
$$

c) The equation of mass diffusion is verified almost everywhere in $\left(0, T^{\prime}\right) \times \Omega$. 
Definition $2 A$ weak solution $(\boldsymbol{u}, \rho)$ of (1) in $\left(0, T^{\prime}\right)$ is said to be a strong solution of Problem (1) in $\left(0, T^{\prime}\right)$ with $T^{\prime}=T<\infty$ or $T^{\prime}=+\infty$ if it satisfies $(\boldsymbol{u}, \rho) \in L^{\infty}\left(0, T^{\prime} ; \boldsymbol{V} \times H_{N}^{2}(\Omega)\right)$,

The rest of the paper is as follows. In Section 2, we study the existence of global weak solutions imposing $\lambda / \mu$ small enough for $2 D$ and $3 D$ domains. In Section 3, we prove the existence and regularity of strong solutions, first for 3D domains and second for the $2 \mathrm{D}$ case, where the main difference between these two cases is that smallness of the data is necessary only for $3 \mathrm{D}$ domains. In Section 4, we study the effects of exponential decay of the external force on the global solution.

\section{Existence of global weak solution}

Theorem 3 Let $\Omega \subset \mathbb{R}^{3}$. Assume $\boldsymbol{u}_{0} \in \boldsymbol{H}, \rho_{0} \in H_{N}^{1}(\Omega)$ verifying (2) and either $\boldsymbol{f} \in L^{2}\left(0, T ; \boldsymbol{L}^{6 / 5}(\Omega)\right)$ if $T<\infty$ or $\boldsymbol{f} \in L^{\infty}\left(0, \infty ; \boldsymbol{L}^{6 / 5}(\Omega)\right)$ if $T=$ $\infty$. Then, if $\lambda / \mu$ is small enough, there exists a weak global solution $(\boldsymbol{u}, \rho)$ of Problem (1) in $(0, T)$ and satisfies

$$
\begin{gathered}
0<m \leq \rho(\boldsymbol{x}, t) \leq M \text { in } Q, \\
\|(\boldsymbol{u}(t), \rho(t))\|_{L^{2} \times H^{1}} \leq C \quad \forall t \geq 0, \\
\forall \gamma>0, \quad e^{-\gamma t} \int_{0}^{t} e^{\gamma s}\|(\boldsymbol{u}(s), \rho(s))\|_{H^{1} \times H^{2}}^{2} d s \leq C, \quad \forall t \geq 0 .
\end{gathered}
$$

Moreover, in the finite time case $(T<\infty)$, one can take $\gamma=0$ in (7).

Proof: Since the Galerkin approximations $\left(\boldsymbol{u}^{k}, \rho^{k}\right)$ will appear in the proof everywhere, there will be no ambiguity in setting $\boldsymbol{u}=\boldsymbol{u}^{k}$ and $\rho=\rho^{k}$. For brevity, we will only prove estimates (5)-(7) in the case $T=+\infty$. 
The maximum principle applied to (4) gives (5).

Working as in [17] we can find the differential inequality (whenever $\lambda / \mu$ small enough)

$$
\frac{d}{d t}\left[\left\|\rho^{1 / 2} \boldsymbol{u}\right\|^{2}+\alpha \lambda\|\nabla \rho\|^{2}\right]+\alpha \lambda^{2}\|\Delta \rho\|^{2}+\mu\|\nabla \boldsymbol{u}\|^{2} \leq C\|\boldsymbol{f}\|_{L^{6 / 5}(\Omega)}^{2}
$$

for a certain $\alpha>0$ (depending on the data).

Let us denote $\varphi_{1}=\left\|\rho^{1 / 2} \boldsymbol{u}\right\|^{2}+\alpha \lambda\|\nabla \rho\|^{2}$ and $\psi_{1}=\alpha \lambda^{2}\|\Delta \rho\|^{2}+\mu\|\nabla \boldsymbol{u}\|^{2}$. Then, (8) is written as

$$
\varphi_{1}^{\prime}+\psi_{1} \leq C\|\boldsymbol{f}\|_{L^{6 / 5}(\Omega)}^{2} \leq C
$$

Using $\varphi_{1} \leq P_{1} \psi_{1}$ where $P_{1}>0$ is a Poincaré constant and multiplying (9) by $e^{\gamma^{*} t}$ for $\gamma^{*}<1 / P_{1}$,

$$
\left(e^{\gamma^{*} t} \varphi_{1}\right)^{\prime}+\left(\frac{1}{P_{1}}-\gamma^{*}\right)\left(e^{\gamma^{*} t} \varphi_{1}\right) \leq C e^{\gamma^{*} t}
$$

which implies

$$
e^{\gamma^{*} t} \varphi_{1}(t) \leq \varphi_{1}(0)+C \int_{0}^{t} e^{\gamma^{*} s} d s \leq \varphi(0)+\frac{C}{\gamma^{*}}\left(e^{\gamma^{*} t-1}\right)
$$

Thus, we obtain $\varphi_{1}(t) \leq e^{-\gamma^{*} t} \varphi_{1}(0)+C\left(1-e^{-\gamma^{*} t}\right) \leq \varphi_{1}(0)+C$ for all $t \geq 0$, hence $\varphi_{1}(t) \leq C, \forall t \geq 0$ and (6) holds.

Getting back to (9), multiplying by $e^{\gamma t}$ for any $\gamma>0$ we get

$$
\left(e^{\gamma t} \varphi_{1}\right)^{\prime}+e^{\gamma t} \psi_{1} \leq C e^{\gamma t}+\gamma e^{\gamma t} \varphi_{1} \leq C e^{\gamma t}
$$

From this last differential inequality is easy to deduce

$$
e^{-\gamma t} \int_{0}^{t} e^{\gamma s} \psi_{1}(s) d s \leq C,
$$

for all $t \geq 0$, hence (7) holds. 


\section{Existence of strong solution and regularity}

This section is divided into two parts: the first part corresponding to 3D Case and the second one to 2D Case. In both cases we study local and global existence of strong solutions and some regularity properties, but the main difference is that for $3 D$ domains some smallness constraints (on data or on the final time) must be imposed, whereas for $2 D$ domains these constraints do not appear.

\subsection{The $3 D$ case}

Theorem 4 Let $\Omega \subset \mathbb{R}^{3}$. Assume $\boldsymbol{u}_{0} \in \boldsymbol{V}$ and $\rho_{0} \in H_{N}^{2}(\Omega)$ satisfying (2).

Case 1: $(T<+\infty)$ Let $\boldsymbol{f} \in L^{2}\left(0, T ; \boldsymbol{L}^{2}(\Omega)\right)$. Then, there exists a unique strong solution $(\boldsymbol{u}, \rho)$ of Problem (1) in $\left(0, T^{*}\right)$ for certain $T^{*} \leq T$, verifying

$$
\begin{gathered}
\|(\boldsymbol{u}(t), \rho(t))\|_{H^{1} \times H^{2}} \leq C, \quad \forall t \in\left[0, T^{*}\right], \\
\int_{0}^{T^{*}}\left\|\left(\boldsymbol{u}(s), \rho(s), \boldsymbol{u}_{t}(s), \rho_{t}(s)\right)\right\|_{H^{2} \times H^{3} \times L^{2} \times H^{1}}^{2} d s \leq C .
\end{gathered}
$$

Moreover, if $\left\|\nabla \boldsymbol{u}_{0}\right\|,\left\|\Delta \rho_{0}\right\|$ are small enough, one can take $T^{*}=T$.

Case 2: $(T=+\infty)$ If $\left\|\nabla \boldsymbol{u}_{0}\right\|,\left\|\Delta \rho_{0}\right\|$ and $\|\boldsymbol{f}\|_{L^{\infty}\left(0, \infty ; L^{2}(\Omega)\right)}$ are small enough, then there exists a unique strong solution $(\boldsymbol{u}, \rho)$ of Problem (1) in $(0, \infty)$ such that

$$
\begin{gathered}
\|(\boldsymbol{u}(t), \rho(t))\|_{H^{1} \times H^{2}} \leq C, \quad \forall t \geq 0, \\
\forall \gamma>0, \quad e^{-\gamma t} \int_{0}^{t} e^{\gamma s}\left\|\left(\boldsymbol{u}(s), \rho(s), \boldsymbol{u}_{t}(s), \rho_{t}(s)\right)\right\|_{H^{2} \times H^{3} \times L^{2} \times H^{1}}^{2} \\
d s \leq C, \quad \forall t \geq 0 .
\end{gathered}
$$

Proof: Again, for brevity in the notation, we denote $\boldsymbol{u}=\boldsymbol{u}^{k}$ and $\rho=\rho^{k}$ and we will see estimates (10)-(11) and (12)-(13).

Taking as test function $\boldsymbol{v}=\boldsymbol{u}_{t}$ into (3), this gives 


$$
\begin{aligned}
\frac{\mu}{2} \frac{d}{d t}\|\nabla \boldsymbol{u}\|^{2}+\left\|\rho^{1 / 2} \boldsymbol{u}_{t}\right\|^{2} & \leq\left|\left(((\rho \boldsymbol{u}-\lambda \nabla \rho) \cdot \nabla) \boldsymbol{u}, \boldsymbol{u}_{t}\right)\right|+\lambda\left|\left((\boldsymbol{u} \cdot \nabla) \nabla \rho, \boldsymbol{u}_{t}\right)\right| \\
& +\lambda^{2}\left|\left(\nabla \cdot\left(\frac{1}{\rho} \nabla \rho \otimes \nabla \rho\right), \boldsymbol{u}_{t}\right)\right|+\left|\left(\rho \boldsymbol{f}, \boldsymbol{u}_{t}\right)\right| .
\end{aligned}
$$

Estimating the terms on the right-hand side of (14), taking into account the Sobolev embedding $H^{1}(\Omega) \hookrightarrow L^{6}(\Omega)$ and the interpolation $\|w\|_{L^{3}(\Omega)} \leq$ $C\|w\|^{1 / 2}\|w\|_{H^{1}(\Omega)}^{1 / 2}$, recalling that $0<m \leq \rho(x, t) \leq M$, one has $([9])$

$$
\begin{aligned}
\frac{\mu}{2} \frac{d}{d t}\|\nabla \boldsymbol{u}\|^{2}+\frac{3 m}{4}\left\|\boldsymbol{u}_{t}\right\|^{2} & \leq C\|\boldsymbol{f}\|^{2}+C\left(\|\nabla \boldsymbol{u}\|^{2}+\|\Delta \rho\|^{2}\right)^{3} \\
& +\delta\|A \boldsymbol{u}\|^{2}+\gamma\|\nabla \Delta \rho\|^{2}
\end{aligned}
$$

being $\gamma$ and $\delta$ positive constants to be chosen later (and $C>0$ is a constant depending on $\gamma$ and $\delta$ ). In order to control the term $\|A \boldsymbol{u}\|^{2}$ which appears in (15), we take as test function $\boldsymbol{v}=A \boldsymbol{u}$ in (3) (that is possible because the spectral basis of $A$ has been considered), getting

$$
\begin{aligned}
\mu\|A \boldsymbol{u}\|^{2}= & -\left(\rho \boldsymbol{u}_{t}, A \boldsymbol{u}\right)-(((\rho \boldsymbol{u}-\lambda \nabla \rho) \cdot \nabla) \boldsymbol{u}, A \boldsymbol{u})-\lambda((\boldsymbol{u} \cdot \nabla) \nabla \rho, A \boldsymbol{u}) \\
& +\lambda^{2}\left(\nabla \cdot\left(\frac{1}{\rho} \nabla \rho \otimes \nabla \rho\right), A \boldsymbol{u}\right)-(\rho \boldsymbol{f}, A \boldsymbol{u}) .
\end{aligned}
$$

The terms on the right-hand side can be estimated in the same way as before.

We only bound the first term

$$
\left|\left(\rho \boldsymbol{u}_{t}, A \boldsymbol{u}\right)\right| \leq M\left\|\boldsymbol{u}_{t}\right\|\|A \boldsymbol{u}\| \leq \frac{M^{2}}{\mu}\left\|\boldsymbol{u}_{t}\right\|^{2}+\frac{\mu}{4}\|A \boldsymbol{u}\|^{2} .
$$

Then, we get the inequality

$$
\frac{\mu}{2}\|A \boldsymbol{u}\|^{2} \leq \frac{M^{2}}{\mu}\left\|\boldsymbol{u}_{t}\right\|^{2}+C\left(\|\nabla \boldsymbol{u}\|^{2}+\|\nabla \boldsymbol{u}\|^{2}\right)^{3}+\gamma\|\nabla \Delta \rho\|^{2}+C\|\boldsymbol{f}\|^{2} .
$$

Multiplying (16) by $\frac{m \mu}{4 M^{2}}$ and adding to (15), one has (choosing $\delta$ small enough) 


$$
\begin{aligned}
\frac{\mu}{2} \frac{d}{d t}\|\nabla \boldsymbol{u}\|^{2}+\frac{m}{2}\left\|\boldsymbol{u}_{t}\right\|^{2}+\frac{m \mu^{2}}{8 M^{2}}\|A \boldsymbol{u}\|^{2} & \leq C\left(\|\Delta \rho\|^{2}+\|\nabla \boldsymbol{u}\|^{2}\right)^{3} \\
& +\gamma\|\nabla \Delta \rho\|^{2}+C\|\boldsymbol{f}\|^{2} .
\end{aligned}
$$

On the other hand, multiplying the density equation (4) by $-\Delta \rho_{t}+\lambda \Delta^{2} \rho$ and integrating by parts, one obtains

$$
\left\|\nabla \rho_{t}\right\|^{2}+\lambda \frac{d}{d t}\|\Delta \rho\|^{2}+\lambda^{2}\|\nabla \Delta \rho\|^{2}=\left(\nabla(\boldsymbol{u} \cdot \nabla \rho),-\nabla \rho_{t}+\lambda \nabla \Delta \rho\right) .
$$

Using the Hölder's and Young's inequalities in the previous equality, it becomes (for arbitrary $\delta>0$ )

$$
\lambda \frac{d}{d t}\|\Delta \rho\|^{2}+\left\|\nabla \rho_{t}\right\|^{2}+\frac{\lambda^{2}}{2}\|\nabla \Delta \rho\|^{2} \leq C\left(\|\nabla \boldsymbol{u}\|^{2}+\|\Delta \rho\|^{2}\right)^{3}+\delta\|A \boldsymbol{u}\|^{2} .
$$

Finally, adding inequalities (18) and (19) and choosing $\gamma$ and $\delta$ small enough, we obtain

$$
\begin{aligned}
& \frac{d}{d t}\left\{\frac{\mu}{2}\|\nabla \boldsymbol{u}\|^{2}+\lambda\|\Delta \rho\|^{2}\right\}+\frac{m}{2}\left\|\boldsymbol{u}_{t}\right\|^{2}+\left\|\nabla \rho_{t}\right\|^{2}+\frac{m \mu^{2}}{8 M^{2}}\|A \boldsymbol{u}\|^{2} \\
& +\frac{\lambda^{2}}{4}\|\nabla \Delta \rho\|^{2} \leq C\left(\|\Delta \rho\|^{2}+\|\nabla \boldsymbol{u}\|^{2}\right)^{3}+C\|\boldsymbol{f}\|^{2} .
\end{aligned}
$$

By setting $\varphi_{2}(t)=\frac{\mu}{2}\|\nabla \boldsymbol{u}(t)\|^{2}+\|\Delta \rho(t)\|^{2}, \chi_{2}(t)=\frac{m}{2}\left\|\boldsymbol{u}_{t}\right\|^{2}+\left\|\nabla \rho_{t}\right\|^{2}$ and $\psi_{2}(t)=\frac{m \mu^{2}}{8 M^{2}}\|A \boldsymbol{u}\|^{2}+\frac{\lambda^{2}}{4}\|\nabla \Delta \rho\|^{2}$, inequality (19) can be written as

$$
\varphi_{2}^{\prime}(t)+\chi_{2}(t)+\psi_{2}(t) \leq C \varphi_{2}^{3}(t)+C\|\boldsymbol{f}(t)\|^{2} .
$$

Thus, using that $\psi_{2} \geq P_{2} \varphi_{2}$ (with $P_{2}>0$ ) and classical results of differential inequalities we shall obtain two results:

Case 1: There exists a time $T^{*} \leq T$ small enough such that $\varphi_{2}(t) \leq C$ for all $t \in\left[0, T^{*}\right]$ and $\int_{0}^{T^{*}}\left(\chi_{2}(s)+\psi_{2}(s)\right) d s \leq C$ (see [11]), hence (10) and (11) hold. 
On the other hand, if $\varphi_{2}(0)$ is sufficiently small, we get $T^{*}=T([19])$.

Case 2: For $\left\|\nabla \boldsymbol{u}_{0}\right\|^{2},\left\|\Delta \rho_{0}\right\|^{2}$ and $\|\boldsymbol{f}\|_{L^{\infty}\left(0, T ; L^{6 / 5}(\Omega)\right)}$ sufficiently small, one obtains $\varphi_{2}(t) \leq C$, hence (12) holds. Getting back to (20) and multiplying by $e^{\gamma t}$, we can get $e^{-\gamma t} \int_{0}^{t} e^{\gamma s}\left(\chi_{2}(s)+\psi_{2}(s)\right) d s \leq C$ for all $t \geq 0$, hence (13) holds.

Corollary 5 Assume hypotheses of Theorem 4, $\boldsymbol{u}_{0} \in \boldsymbol{V} \cap \boldsymbol{H}^{2}(\Omega)$ and $\rho_{0} \in$ $H_{N}^{3}(\Omega)$.

Case 1: $(T<+\infty)$ Let $\boldsymbol{f} \in L^{2}\left(0, T ; \boldsymbol{H}^{1}(\Omega)\right)$ and $\boldsymbol{f}_{t}$ $\in L^{2}\left(0, T ; \boldsymbol{L}^{6 / 5}(\Omega)\right)$. Then, the unique strong solution $(\boldsymbol{u}, \rho)$ of Problem (1) in $\left(0, T^{*}\right)$ for certain $T^{*} \leq T$ given in Theorem 4 verifies the additional estimates:

$$
\begin{gathered}
\left\|\left(\boldsymbol{u}(t), \rho(t), \boldsymbol{u}_{t}(t), \rho_{t}(t)\right)\right\|_{H^{2} \times H^{3} \times L^{2} \times H^{1}} \leq C, \quad \forall t \in\left[0, T^{*}\right], \\
\int_{0}^{T^{*}}\left\|\left(\boldsymbol{u}(s), \rho(s), \boldsymbol{u}_{t}(s), \rho_{t}(s)\right)\right\|_{H^{3} \times H^{4} \times H^{1} \times H^{2}}^{2} \leq C .
\end{gathered}
$$

Moreover, if $\rho_{0} \in H_{N}^{4}(\Omega)$

$$
\begin{gathered}
\left\|\left(\sigma^{1 / 2}(t) \boldsymbol{u}(t), \rho(t), \sigma^{1 / 2} \boldsymbol{u}_{t}(t), \rho_{t}(t)\right)\right\|_{H^{3} \times H^{4} \times H^{1} \times H^{2}} \leq C, \quad \forall t \in\left[0, T^{*}\right], \\
\int_{0}^{T^{*}}\left\|\left(\sigma^{1 / 2} \boldsymbol{u}, \rho, \sigma^{1 / 2} \boldsymbol{u}_{t}, \rho_{t}, \sigma^{1 / 2} \boldsymbol{u}_{t t}, \rho_{t t}\right)\right\|_{H^{4} \times H^{5} \times H^{2} \times H^{3} \times L^{2} \times H^{1}}^{2} \leq C
\end{gathered}
$$

where $\sigma(t)=\min \{1, t\}$.

Case 2: $(T=+\infty)$ Let $\boldsymbol{f} \in L^{\infty}\left(0, \infty ; \boldsymbol{H}^{1}(\Omega)\right)$ and $\boldsymbol{f}_{t} \in L^{\infty}\left(0, \infty ; \boldsymbol{L}^{6 / 5}(\Omega)\right)$. Then, the unique strong solution $(\boldsymbol{u}, \rho)$ of Problem (1) in $(0, \infty)$ given in Theorem 4 , verifies

$$
\left\|\left(\boldsymbol{u}(t), \rho(t), \boldsymbol{u}_{t}(t), \rho_{t}(t)\right)\right\|_{\boldsymbol{H}^{2} \times H^{3} \times \boldsymbol{L}^{2} \times H^{1}} \leq C, \quad \forall t \geq 0,
$$


$\forall \gamma>0, \quad e^{-\gamma t} \int_{0}^{t} e^{-\gamma s}\left\|\left(\boldsymbol{u}(s), \rho(s), \boldsymbol{u}_{t}(s), \rho_{t}(s)\right)\right\|_{H^{3} \times H^{4} \times H^{1} \times H^{2}}^{2} \leq C$.

Moreover, if $\rho_{0} \in H_{N}^{4}(\Omega)$

$$
\begin{aligned}
& \left\|\left(\sigma^{1 / 2}(t) \boldsymbol{u}(t), \rho(t), \sigma^{1 / 2} \boldsymbol{u}_{t}(t), \rho_{t}(t)\right)\right\|_{\boldsymbol{H}^{3} \times H^{4} \times \boldsymbol{H}^{1} \times H^{2}} \leq C, \quad \forall t \geq 0, \\
& \forall \gamma>0, \quad e^{-\gamma t} \int_{0}^{t} e^{\gamma s}\left\|\left(\sigma^{1 / 2} \boldsymbol{u}, \rho, \sigma^{1 / 2} \boldsymbol{u}_{t}, \rho_{t}, \sigma^{1 / 2} \boldsymbol{u}_{t t}, \rho_{t t}\right)\right\|_{H^{4} \times H^{5} \times H^{2} \times H^{3} \times L^{2} \times H^{1}}^{2} \\
& \leq C, \forall t \geq 0 .
\end{aligned}
$$

Proof: Firstly, we are going to improve the a priori estimates obtained in the previous Theorem for $\left(\boldsymbol{u}_{t}, \rho_{t}\right)$. As a consequence, we will improve the estimates obtained for $(\boldsymbol{u}, \rho)$ in $D(A)$ and $H^{3}(\Omega)$ norms respectively.

By differentiating the density equation (4) with respect to $t$ and taking the inner product of $L^{2}(\Omega)$ with the term $\Delta \rho_{t}$ and integrating by parts

$$
\begin{aligned}
\frac{1}{2} \frac{d}{d t}\left\|\nabla \rho_{t}\right\|^{2}+\lambda\left\|\Delta \rho_{t}\right\|^{2} & \leq\left|\left(\nabla \boldsymbol{u}_{t} \cdot \nabla \rho, \nabla \rho_{t}\right)\right|+\left|\left(\boldsymbol{u}_{t} \cdot \nabla^{2} \rho, \nabla \rho_{t}\right)\right| \\
& +\left|\left(\nabla \boldsymbol{u} \cdot \nabla \rho_{t}, \nabla \rho_{t}\right)\right|+\left|\left(\boldsymbol{u} \cdot \nabla^{2} \rho_{t}, \nabla \rho_{t}\right)\right| .
\end{aligned}
$$

In virtue of the bounds for $\|\nabla \boldsymbol{u}\|$ and $\|\Delta \rho\|$ obtained in Theorem 4, the terms on the right-hand side can be estimated using the interpolation inequalities $\left\|\nabla^{2} \rho\right\|_{3} \leq C\|\Delta \rho\|^{1 / 2}\|\nabla \Delta \rho\|^{1 / 2}$ and $\|\nabla \rho\|_{4} \leq C\left\|\Delta \rho_{t}\right\|^{1 / 4}\left\|\nabla \rho_{t}\right\|^{3 / 4}$, the Sobolev imbedding $H^{1}(\Omega) \hookrightarrow L^{6}(\Omega)$ and Young's inequality, getting

$$
\frac{1}{2} \frac{d}{d t}\left\|\nabla \rho_{t}\right\|^{2}+\frac{3 \lambda}{4}\left\|\Delta \rho_{t}\right\|^{2} \leq \gamma\left\|\nabla \boldsymbol{u}_{t}\right\|^{2}+C\left\|\nabla \rho_{t}\right\|^{2},
$$

for any $\gamma>0$ (with $C$ depending on $\gamma$ ). 
Now, computing the derivative of system (3) with respect to $t$, taking as test function $\boldsymbol{v}=\boldsymbol{u}_{t}$ and take into account equation (4), one gets

$$
\begin{aligned}
& \frac{1}{2} \frac{d}{d t}\left\|\rho^{1 / 2} \boldsymbol{u}_{t}\right\|^{2}+\mu\left\|\nabla \boldsymbol{u}_{t}\right\|^{2} \\
& =-\left(\rho_{t}(\boldsymbol{u} \cdot \nabla) \boldsymbol{u}, \boldsymbol{u}_{t}\right)-\left(\rho\left(\boldsymbol{u}_{t} \cdot \nabla\right) \boldsymbol{u}, \boldsymbol{u}_{t}\right)+\left(\rho_{t} \boldsymbol{f}, \boldsymbol{u}_{t}\right)+\left(\rho \boldsymbol{f}_{t}, \boldsymbol{u}_{t}\right) \\
& +\lambda\left\{\left(\left(\nabla \rho_{t} \cdot \nabla\right) \boldsymbol{u}, \boldsymbol{u}_{t}\right)+\left(\left(\boldsymbol{u}_{t} \cdot \nabla\right) \nabla \rho, \boldsymbol{u}_{t}\right)\right\}+\lambda\left((\boldsymbol{u} \cdot \nabla) \nabla \rho_{t}, \boldsymbol{u}_{t}\right) \\
& +\lambda^{2}\left(\frac{\rho_{t}}{\rho^{2}} \nabla \rho \otimes \nabla \rho, \nabla \boldsymbol{u}_{t}\right)-\lambda^{2}\left(\frac{1}{\rho} \nabla \rho_{t} \otimes \nabla \rho, \nabla \boldsymbol{u}_{t}\right)-\lambda^{2}\left(\frac{1}{\rho} \nabla \rho \otimes \nabla \rho_{t}, \nabla \boldsymbol{u}_{t}\right) .
\end{aligned}
$$

We observe that the terms $\left(\rho_{t}(\boldsymbol{u} \cdot \nabla) \boldsymbol{u}, \boldsymbol{u}_{t}\right)$ and $\left(\rho\left(\boldsymbol{u}_{t} \cdot \nabla\right) \boldsymbol{u}, \boldsymbol{u}_{t}\right)$ can be bounded as in the Navier-Stokes case ([11]) and the bounds for the terms $\lambda\left(\left(\nabla \rho_{t}\right.\right.$. $\left.\nabla) \boldsymbol{u}, \boldsymbol{u}_{t}\right)$ and $\lambda\left(\left(\boldsymbol{u}_{t} \cdot \nabla\right) \nabla \rho, \boldsymbol{u}_{t}\right)$ are similar. The other terms are estimated as follows

$$
\begin{aligned}
&\left|\left(\rho_{t}(\boldsymbol{u} \cdot \nabla) \boldsymbol{u}, \boldsymbol{u}_{t}\right)\right| \leq\left|\left(\boldsymbol{u} \cdot \nabla \rho(\boldsymbol{u} \cdot \nabla) \boldsymbol{u}, \boldsymbol{u}_{t}\right)\right|+\lambda \mid\left(\Delta \rho(\boldsymbol{u} \cdot \nabla) \boldsymbol{u}, \boldsymbol{u}_{t} \mid \quad\right. \text { (using eq. (4)) } \\
& \leq\|\boldsymbol{u}\|_{\infty}\|\nabla \rho\|_{L^{6}}\|\boldsymbol{u}\|_{L^{6}}\|\nabla \boldsymbol{u}\|\left\|\boldsymbol{u}_{t}\right\|_{L^{6}}+\|\Delta \rho\|\|\boldsymbol{u}\|_{L^{\infty}}\|\nabla \boldsymbol{u}\|_{L^{3}}\left\|\boldsymbol{u}_{t}\right\|_{L^{6}} \\
& \leq C\|A \boldsymbol{u}\|^{2}+\gamma\left\|\nabla \boldsymbol{u}_{t}\right\|^{2} \\
&\left|\left(\rho_{t} \boldsymbol{f}, \boldsymbol{u}_{t}\right)\right| \leq\|\boldsymbol{f}\|\left\|\rho_{t}\right\|_{L^{3}}\left\|\boldsymbol{u}_{t}\right\|_{L^{6}} \leq C\|\boldsymbol{f}\|^{2}\left\|\nabla \rho_{t}\right\|^{2}+\gamma\left\|\nabla \boldsymbol{u}_{t}\right\|^{2} \\
&\left(\frac{\rho_{t}}{\rho} \nabla \rho \otimes \nabla \rho, \nabla \boldsymbol{u}_{t}\right) \leq \frac{C}{m}\left\|\nabla \rho_{t}\right\|\|\Delta \rho\|^{2}\left\|\nabla \boldsymbol{u}_{t} \leq C\right\| \nabla \rho_{t}\left\|^{2}+\gamma\right\| \nabla \boldsymbol{u}_{t} \|^{2} \\
&\left(\frac{1}{\rho} \nabla \rho_{t} \otimes \nabla \rho, \nabla \boldsymbol{u}_{t}\right)+\left(\frac{1}{\rho} \nabla \rho \otimes \nabla \rho_{t}, \nabla \boldsymbol{u}_{t}\right) \leq \frac{C}{m}\left\|\nabla \rho_{t}\right\|_{L^{3}}\|\nabla \rho\|_{L^{6}}\left\|\nabla \boldsymbol{u}_{t}\right\| \\
& \leq C\left\|\nabla \rho_{t}\right\|^{2}+\varepsilon\left\|\Delta \rho_{t}\right\|^{2} \\
&+\gamma\left\|\nabla \boldsymbol{u}_{t}\right\|^{2}
\end{aligned}
$$

Thus, we obtain the differential inequality (choosing $\gamma$ sufficiently small)

$$
\begin{aligned}
\frac{1}{2} \frac{d}{d t}\left\|\rho^{1 / 2} \boldsymbol{u}_{t}\right\|^{2}+\frac{3 \mu}{4}\left\|\nabla \boldsymbol{u}_{t}\right\|^{2} & \leq C\left(\|\boldsymbol{f}\|^{2}\left\|\nabla \rho_{t}\right\|^{2}+\left\|\boldsymbol{f}_{t}\right\|_{L^{6 / 5}(\Omega)}^{2}\right) \\
& +C\left\|\boldsymbol{u}_{t}\right\|^{2}+C\left\|\nabla \rho_{t}\right\|^{2}+\varepsilon\left\|\Delta \rho_{t}\right\|^{2} .
\end{aligned}
$$

Adding this inequality with (29), choosing suitable $\gamma$ and $\varepsilon$, and taking the notation $\varphi_{3}=\left\|\rho^{1 / 2} \boldsymbol{u}_{t}\right\|^{2}+\left\|\nabla \rho_{t}\right\|^{2}$ and $\psi_{3}=\mu\left\|\nabla \boldsymbol{u}_{t}\right\|^{2}+\lambda\left\|\Delta \rho_{t}\right\|^{2}$, one has

$$
\varphi_{3}^{\prime}+\psi_{3} \leq C\|\boldsymbol{f}\|^{2} \varphi_{3}+C\left\|\boldsymbol{f}_{t}\right\|_{L^{6 / 5}(\Omega)}^{2}+C \varphi_{3} .
$$


Case 1: Using Gronwall's Lemma in (30), since $\varphi_{3}(0) \leq C$ (in fact $\left\|\boldsymbol{u}_{t}(0)\right\| \leq$ $C\left(\left\|\boldsymbol{u}_{0}\right\|_{H^{2}(\Omega)},\left\|\rho_{0}\right\|_{H_{N}^{3}(\Omega)},\|\boldsymbol{f}(0)\|\right)$ and $\left.\left\|\nabla \rho_{t}(0)\right\| \leq C\left(\left\|\boldsymbol{u}_{0}\right\|_{H^{2}(\Omega)},\left\|\rho_{0}\right\|_{H_{N}^{3}(\Omega)}\right)\right)$ we obtain $\varphi_{3}(t) \leq C$ for all $t \in\left[0, T^{*}\right]$ and $\int_{0}^{T^{*}} \psi_{3}(s) d s \leq C$, hence $(21)$ and (22) for $\left(\boldsymbol{u}_{t}, \rho_{t}\right)$ hold.

Case 2: Multiplying by $e^{\gamma t}$ in (30) (for any $\gamma>0$ ) and integrating from 0 to $t$, we get

$$
\begin{aligned}
\varphi_{3}(t)+e^{-\gamma t} \int_{0}^{t} e^{\gamma \tau} \psi_{3}(\tau) d \tau & \leq \varphi_{3}(0)+C e^{-\gamma t} \int_{0}^{t} e^{-\gamma \tau}\left\|\boldsymbol{f}_{t}(\tau)\right\|_{L^{6 / 5}(\Omega)}^{2} d \tau \\
& +C e^{-\gamma t} \int_{0}^{t} e^{-\gamma \tau} \varphi_{3}(\tau) d \tau
\end{aligned}
$$

Applying $e^{-\gamma t} \int_{0}^{t} e^{-\gamma \tau} \varphi_{3}(\tau) \tau \leq C e^{-\gamma t} \int_{0}^{t} e^{-\gamma \tau} \chi_{2}(\tau) \tau \leq C$, we get (25) and (26) for $\left(\boldsymbol{u}_{t}, \rho_{t}\right)$.

Now, we improve the regularity in time for $D(A)$ and $H_{N}^{3}(\Omega)$ norms for the velocity and density respectively. Multiplying (4) by $\Delta^{2} \rho$, integrating over $\Omega$ and integrating by parts,

$$
\frac{\lambda}{2}\|\nabla \Delta \rho\|^{2} \leq C\left\|\nabla \rho_{t}\right\|^{2}+C\left(\|\nabla \boldsymbol{u}\|^{2}+\|\Delta \rho\|^{2}\right)^{3}+\delta\|A \boldsymbol{u}\|^{2}
$$

for any $\delta>0$. Adding (16) and (32), and taking suitable $\gamma$ and $\delta$, one obtains the inequality

$$
\mu\|A \boldsymbol{u}\|^{2}+\lambda\|\nabla \Delta \rho\|^{2} \leq C\left\|\boldsymbol{u}_{t}\right\|^{2}+C\left\|\nabla \rho_{t}\right\|^{2}+C\left(\|\nabla \boldsymbol{u}\|^{2}+\|\Delta \rho\|^{2}\right)^{3}
$$

From this last expression and the above estimates is easy to deduce (21) in Case 1 or $(25)$ in Case 2, for $(\rho, \boldsymbol{u})$. 
Now, we want to obtain better estimates in space for the velocity. By taking $A^{2} \boldsymbol{u}$ as test function in (3), one has

$$
\begin{aligned}
\mu\left\|A^{3 / 2} \boldsymbol{u}\right\| & \leq\left\|A^{1 / 2}\left(\rho \boldsymbol{u}_{t}\right)\right\|+\left\|A^{1 / 2}(((\rho \boldsymbol{u}-\lambda \nabla \rho) \cdot \nabla) \boldsymbol{u})\right\| \\
& +\left\|A^{1 / 2}(\rho \boldsymbol{f})\right\|+\lambda\left\|A^{1 / 2}((\boldsymbol{u} \cdot \nabla) \nabla \rho)\right\| \\
& +\lambda^{2} \mid A^{1 / 2}\left(\nabla \cdot\left(\frac{1}{\rho}(\nabla \rho \otimes \nabla \rho)\right) \mid\right.
\end{aligned}
$$

hence, estimating on the right-hand side one has

$$
\left\|A^{3 / 2} \boldsymbol{u}\right\| \leq C\left\|\nabla \boldsymbol{u}_{t}\right\|+C
$$

hence (22) in Case 1 or (26) in Case 2, for $\boldsymbol{u}$, holds.

Analogously for the density. Applying the operator $\Delta$ to (4) and taking the inner product by $-\Delta^{2} \rho$, one gets

$$
\begin{aligned}
\frac{\lambda}{2}\left\|\Delta^{2} \rho\right\|^{2} & =\left\|\Delta \rho_{t}+\Delta \boldsymbol{u} \cdot \nabla \rho+\boldsymbol{u} \cdot \Delta \nabla \rho\right\|^{2} \\
& \leq C\left(\left\|\Delta \rho_{t}\right\|^{2}+\|\Delta \boldsymbol{u}\|^{2}\|\nabla \rho\|_{\infty}^{2}+\|\boldsymbol{u}\|_{\infty}^{2}\|\nabla \Delta \rho\|^{2}\right) \\
& \leq C\left(\left\|\Delta \rho_{t}\right\|^{2}+\|A \boldsymbol{u}\|^{2}\|\rho\|_{H_{N}^{3}}^{2}\right) \leq C\left(\left\|\Delta \rho_{t}\right\|^{2}+1\right) .
\end{aligned}
$$

Therefore, we conclude (22) in Case $\mathbf{1}$ or (26) in Case 2, for $\rho$.

In what follow, we suppose that $\rho_{0} \in H_{N}^{4}(\Omega)$. By differentiating the approximate momentum system (3) with respect to $t$ and considering $\boldsymbol{v}=\boldsymbol{u}_{t t}$ as test function, we have

$$
\begin{aligned}
\left\|\rho^{1 / 2} \boldsymbol{u}_{t t}\right\|^{2}+\frac{\mu}{2} \frac{d}{d t}\left\|\nabla \boldsymbol{u}_{t}\right\|^{2}= & \left.-([(\rho \boldsymbol{u}-\lambda \nabla \rho) \cdot \nabla) \boldsymbol{u}]_{t}, \boldsymbol{u}_{t t}\right) \\
& +\lambda\left([(\boldsymbol{u} \cdot \nabla) \nabla \rho]_{t}, \boldsymbol{u}_{t t}\right) \\
& -\lambda^{2}\left(\left[\nabla \cdot\left(\frac{1}{\rho^{k}} \nabla \rho^{k} \otimes \nabla \rho^{k}\right)\right]_{t}, \boldsymbol{u}_{t t}\right)+\left(\rho^{k} \boldsymbol{f}, \boldsymbol{u}_{t t}\right) .
\end{aligned}
$$

Thus, bounding on the right-hand side, one obtains the following differential inequality $([9])$

$$
\begin{aligned}
\left\|\rho^{1 / 2} \boldsymbol{u}_{t t}\right\|^{2}+\mu \frac{d}{d t}\left\|\nabla \boldsymbol{u}_{t}\right\|^{2} \leq & C\left\|\nabla \boldsymbol{u}_{t}\right\|^{2}+C\left\|\Delta \rho_{t}\right\|^{2}+C\|\boldsymbol{f}\|_{\boldsymbol{H}^{1}}^{2} \\
& +C\left\|\boldsymbol{f}_{t}\right\|^{2}+C .
\end{aligned}
$$


Analogously, for the approximate density, we get

$$
\left\|\nabla \rho_{t t}\right\|^{2}+\lambda \frac{d}{d t}\left\|\Delta \rho_{t}\right\|^{2} \leq C\left\|\nabla \boldsymbol{u}_{t}\right\|^{2}+C\left\|\Delta \rho_{t}\right\|^{2}
$$

Case 1: Multiplying (36) by $\sigma(t)$, where $\sigma(t)=\min \{t, 1\}$, we have

$$
\begin{aligned}
& \sigma(s)\left\|\rho^{1 / 2} \boldsymbol{u}_{t t}(s)\right\|^{2}+\mu \frac{d}{d t}\left(\sigma(s)\left\|\nabla \boldsymbol{u}_{t}(s)\right\|^{2}\right)-\mu \sigma^{\prime}(s)\left\|\nabla \boldsymbol{u}_{t}(s)\right\|^{2} \\
& \leq C \sigma(s)\left(\left\|\nabla \boldsymbol{u}_{t}\right\|^{2}+\left\|\Delta \rho_{t}\right\|^{2}+\|\boldsymbol{f}\|_{\boldsymbol{H}^{1}}^{2}+\left\|\boldsymbol{f}_{t}\right\|^{2}+1\right) .
\end{aligned}
$$

Integrating (38) from $\varepsilon$ to $t$, with $0<\varepsilon<t$ and using that $\sigma(t) \leq 1$ and $\sigma^{\prime}(t) \leq 1$ a.e. in $[0, t]$, we have

$$
\begin{aligned}
& \sigma(t) \mu\left\|\nabla \boldsymbol{u}_{t}(t)\right\|^{2}+m \int_{\varepsilon}^{t} \sigma(s)\left\|\boldsymbol{u}_{t t}(s)\right\|^{2} d s \leq \mu \sigma(\varepsilon)\left\|\nabla \boldsymbol{u}_{t}(\varepsilon)\right\|^{2} \\
& +C \int_{\varepsilon}^{t}\left(\|\boldsymbol{f}(s)\|_{\boldsymbol{H}^{1}}^{2}+\left\|\boldsymbol{f}_{t}(s)\right\|^{2}+\left\|\nabla \boldsymbol{u}_{t}\right\|^{2}+\left\|\Delta \rho_{t}\right\|^{2}+1\right) d s
\end{aligned}
$$

Since $\nabla \boldsymbol{u}_{t} \in L^{2}(0, t)$, we can choose a sequence $\left(\varepsilon_{n}\right)$ such that $0<\varepsilon_{n}<1$ for all $n \geq 1$ and $\sigma\left(\varepsilon_{n}\right)\left\|\nabla \boldsymbol{u}_{t}\left(\varepsilon_{n}\right)\right\|^{2} \rightarrow 0$ as $\varepsilon_{n} \rightarrow 0$. Then, taking $\varepsilon_{n} \rightarrow 0$, we have

$$
\begin{aligned}
& \mu \sigma(t)\left\|\nabla \boldsymbol{u}_{t}(t)\right\|^{2}+m \int_{0}^{t} \sigma(s)\left\|\boldsymbol{u}_{t t}(s)\right\|^{2} d s \\
& \leq C \int_{0}^{t}\left(\|\boldsymbol{f}(s)\|_{H^{1}}^{2}+\left\|\boldsymbol{f}_{t}(s)\right\|^{2}+\left\|\nabla \boldsymbol{u}_{t}\right\|^{2}+\left\|\Delta \rho_{t}\right\|^{2}+1\right) d s .
\end{aligned}
$$

On the other hand, working for the density as for the velocity, we shall obtain

$$
\begin{aligned}
& \lambda\left\|\Delta \rho_{t}(t)\right\|^{2}+\int_{0}^{t}\left\|\nabla \rho_{t t}(s)\right\|^{2} d s \\
& \leq \lambda\left\|\Delta \rho_{t}(0)\right\|^{2}+C \int_{0}^{t}\left(\left\|\nabla \boldsymbol{u}_{t}\right\|^{2}+\left\|\Delta \rho_{t}\right\|^{2}+1\right) d s .
\end{aligned}
$$

It is easy to bound $\left\|\Delta \rho_{t}(0)\right\| \leq C\left(\left\|\boldsymbol{u}_{0}\right\|_{H^{2}},\left\|\rho_{0}\right\|_{H^{4}}\right)$.

Then, we can conclude using the a priori estimates $\left(\boldsymbol{u}_{t}, \rho_{t}\right) \in L^{2}\left(0, T^{*} ; \boldsymbol{V} \times\right.$ $\left.H^{2}(\Omega)\right)$ that $\sigma^{1 / 2} \boldsymbol{u}_{t} \in L^{\infty}\left(0, T^{*} ; \boldsymbol{V}\right), \quad \sigma^{1 / 2} \boldsymbol{u}_{t t} \in L^{2}\left(0, T^{*} ; \boldsymbol{H}\right), \quad \rho_{t} \in$ $L^{\infty}\left(0, T^{*} ; H_{N}^{2}(\Omega)\right)$ and $\rho_{t t} \in L^{2}\left(0, T^{*} ; H_{N}^{1}(\Omega)\right)$. As well, from (34) and (35) and previous estimates, we infer $(\boldsymbol{u}, \rho) \in L^{\infty}\left(0, T^{*} ; H^{3}(\Omega) \times H^{4}(\Omega)\right)$. 
Now, we want to obtain the following estimate

$$
\int_{0}^{T^{*}}\left(\sigma(s)\left\|A \boldsymbol{u}_{t}(s)\right\|^{2}+\left\|\nabla \Delta \rho_{t}(s)\right\|^{2}\right) d s \leq C .
$$

Indeed, this bound can be obtained by taking $\boldsymbol{v}=A \boldsymbol{u}_{t}$ as test function in the time derivative of the momentum system and applying the gradient operator $\nabla$ in the time derivative of the density equation multiplied by $\nabla \Delta \rho_{t}$ and then bounding the right-hand side of both equalities having in consideration the previous estimates.

On the other hand, taking $\boldsymbol{v}=A^{3} \boldsymbol{u}_{t}$ as test function in (3) and bounding the right-hand side using the estimates obtained up to the moment, we get

$$
\left\|A^{2} \boldsymbol{u}\right\|^{2} \leq C\left(\left\|A \boldsymbol{u}_{t}\right\|^{2}+1\right)
$$

As a consequence, $\sigma^{1 / 2} \boldsymbol{u} \in L^{2}\left(0, T^{*} ; H^{4}(\Omega)\right)$. Analogously for the density, we can obtain $\rho \in L^{2}\left(0, T^{*} ; H^{5}(\Omega)\right)$.

Case 2: Working as in Case 1 changing the weight $\sigma(t)$ for $\widetilde{\sigma}(t)=e^{\gamma t} \sigma(t)$ and using that $\tilde{\sigma}(t) \leq e^{\gamma t}$ and $\tilde{\sigma}^{\prime}(t) \leq C e^{\gamma t}$, one has

$$
\begin{aligned}
& \mu \widetilde{\sigma}(t)\left\|\nabla \boldsymbol{u}_{t}(t)\right\|^{2}+\int_{\varepsilon}^{t} \tilde{\sigma}(s)\left\|\boldsymbol{u}_{t t}(s)\right\|^{2} d s \leq \widetilde{\sigma}(\varepsilon)\left\|\nabla \boldsymbol{u}_{t}(\varepsilon)\right\|^{2} \\
& +C \int_{\varepsilon}^{t} e^{\gamma s}\left(\|\boldsymbol{f}(s)\|_{\boldsymbol{H}^{1}}^{2}+\left\|\boldsymbol{f}_{t}(s)\right\|^{2}+\left\|\nabla \boldsymbol{u}_{t}\right\|^{2}+\left\|\Delta \rho_{t}\right\|^{2}+1\right) d s .
\end{aligned}
$$

Choosing a special sequence $\left(\varepsilon_{n}\right) \rightarrow 0$ such that $\widetilde{\sigma}\left(\varepsilon_{n}\right)\left\|\nabla \boldsymbol{u}_{t}\left(\varepsilon_{n}\right)\right\|^{2} \rightarrow 0$ as $n \rightarrow \infty$ and taking limit, one obtains

$$
\begin{aligned}
& \mu \sigma(t)\left\|\nabla \boldsymbol{u}_{t}(t)\right\|^{2}+\int_{0}^{t} \sigma(s)\left\|\boldsymbol{u}_{t t}(s)\right\|^{2} d s \\
& \leq C e^{-\gamma t} \int_{0}^{t} e^{\gamma s}\left(\|\boldsymbol{f}(s)\|_{\boldsymbol{H}^{1}}^{2}+\left\|\boldsymbol{f}_{t}(s)\right\|^{2}+\left\|\nabla \boldsymbol{u}_{t}\right\|^{2}+\left\|\Delta \rho_{t}\right\|^{2}+1\right) d s .
\end{aligned}
$$

from which one deduces $\sigma^{1 / 2} \boldsymbol{u}_{t} \in L^{\infty}\left(0, \infty ; \boldsymbol{H}^{1}\right)$ and $\sigma^{1 / 2} \boldsymbol{u}_{t t} \in L^{2}\left(0, \infty ; \boldsymbol{L}^{2}(\Omega)\right)$, thanks to the estimate $e^{-\gamma t} \int_{0}^{t} e^{\gamma \tau} \psi_{3}(\tau) d \tau \leq C$. 
Multiplying (37) by $e^{\gamma t}$, we can get

$$
\begin{aligned}
& \lambda\left\|\Delta \rho_{t}(t)\right\|^{2}+e^{-\gamma t} \int_{0}^{t} e^{\gamma s}\left\|\nabla \rho_{t t}(s)\right\|^{2} d s \\
& \leq \lambda e^{-\gamma t}\left\|\Delta \rho_{t}(0)\right\|^{2}+C e^{-\gamma t} \int_{0}^{t} e^{\gamma s}\left(\left\|\nabla \boldsymbol{u}_{t}\right\|^{2}+\left\|\Delta \rho_{t}\right\|^{2}+1\right) d s .
\end{aligned}
$$

Using $e^{-\gamma t} \int_{0}^{t} e^{\gamma \tau} \psi_{3}(\tau) d \tau \leq C$, we deduce that $\rho_{t} \in L^{\infty}\left(0, \infty ; H^{2}(\Omega)\right)$ and $\rho_{t t} \in L^{2}\left(0, \infty ; H^{1}(\Omega)\right)$.

In the similar manner as in Case 1, we deduce $\left(\sigma^{1 / 2} \boldsymbol{u}, \rho\right) \in L_{l o c}^{2}\left(0, \infty ; H^{4}(\Omega) \times\right.$ $\left.H^{5}(\Omega)\right)$ and

$$
\forall \gamma>0, \quad e^{-\gamma t} \int_{0}^{T} e^{\gamma s}\left(\sigma(s)\left\|A^{2} \boldsymbol{u}(s)\right\|^{2}+\left\|\nabla \Delta^{2} \rho(s)\right\|^{2}\right) d s \leq C, \quad \forall t \geq 0 .
$$

\subsection{The $2 D$ case}

Secchi needed to introduce in [18] the condition $\lambda / \mu$ sufficiently small in order to obtain weak estimates. Now, we impose that condition in the following theorem in the case of $2 \mathrm{D}$ domains, obtaining regularity of weak solutions without constraints on data or on the final time .

Theorem 6 Let $\Omega \subset \mathbb{R}^{2}$. Assume $\boldsymbol{u}_{0} \in \boldsymbol{V}, \rho_{0} \in H_{N}^{2}(\Omega)$ verifying (2) and $\lambda / \mu$ is small enough.

Case 1: $(T<+\infty)$ Let $\boldsymbol{f} \in L^{2}\left(0, T ; \boldsymbol{L}^{2}(\Omega)\right)$. Then, there exists a unique strong solution $(\boldsymbol{u}, \rho)$ of Problem (1) in $(0, T)$, verifying

$$
\begin{gathered}
\|(\boldsymbol{u}(t), \rho(t))\|_{H^{1} \times H^{2}} \leq C, \quad \forall t \in[0, T], \\
\int_{0}^{T}\left\|\left(\boldsymbol{u}(s), \rho(s), \boldsymbol{u}_{t}(s), \rho_{t}(s)\right)\right\|_{H^{2} \times H^{3} \times L^{2} \times H^{1}}^{2} \leq C .
\end{gathered}
$$


Case 2: $(T=+\infty)$ Let $\boldsymbol{f} \in L^{\infty}\left(0, \infty ; L^{2}(\Omega)\right)$. Then, there exists a unique strong solution $(\boldsymbol{u}, \rho)$ of Problem (1) in $(0, \infty)$ such that

$$
\|(\boldsymbol{u}(t), \rho(t))\|_{H^{1} \times H^{2}} \leq C, \quad \forall t \geq 0,
$$

$$
\begin{aligned}
& \forall \gamma>0, e^{-\gamma t} \int_{0}^{t} e^{\gamma s}\left\|\left(\boldsymbol{u}(s), \rho(s), \boldsymbol{u}_{t}(s), \rho_{t}(s)\right)\right\|_{H^{2} \times H^{3} \times L^{2} \times H^{1}}^{2} \leq C, \\
& \forall t \geq 0 .
\end{aligned}
$$

Proof: Again there will be no ambiguity in setting $\boldsymbol{u}=\boldsymbol{u}^{k}$ and $\rho=\rho^{k}$. From Theorem 3 (notice that we can choose $\boldsymbol{f} \in L^{2}\left(0, T ; L^{p}(\Omega)\right.$ if $T<\infty$ or $\boldsymbol{f} \in L^{\infty}\left(0, \infty ; L^{p}(\Omega)\right)$ if $T=\infty$ with $p>1$, since we are considering $\left.\Omega \subset \mathbb{R}^{2}\right)$, we can deduce the following estimates:

$$
\begin{gathered}
0<m \leq \rho(\boldsymbol{x}, t) \leq M \text { in } Q, \\
\|(\boldsymbol{u}(t), \rho(t))\|_{L^{2} \times H^{1}} \leq C \quad \forall t \in\left[0, T^{*}\right], \\
\forall \gamma>0, \quad e^{-\gamma t} \int_{0}^{t} e^{\gamma s}\|(\boldsymbol{u}(s), \rho(s))\|_{H^{1} \times H^{2}}^{2} d s \leq C, \quad \forall t \geq 0 .
\end{gathered}
$$

In the finite time case $(T<\infty)$, one can take $\gamma=0$ in (45).

On the other hand, by working as in Theorem 4 and taking into account the Gagliardo-Nirenberg inequality $\|w\|_{L^{4}(\Omega)} \leq C\|w\|^{1 / 2}\|w\|_{H^{1}}$ and (44), we get

$$
\varphi_{2}^{\prime}(t)+\chi_{2}(t)+\psi_{2}(t) \leq C \varphi_{2}^{2}(t)+C\|\boldsymbol{f}(t)\|^{2} .
$$

where $\varphi_{2}(t)=\frac{\mu}{2}\|\nabla \boldsymbol{u}(t)\|^{2}+\|\Delta \rho(t)\|^{2}, \chi_{2}(t)=\frac{m}{2}\left\|\boldsymbol{u}_{t}\right\|^{2}+\left\|\nabla \rho_{t}\right\|^{2}$ and $\psi_{2}(t)=$ $\frac{m \mu^{2}}{8 M^{2}}\|A \boldsymbol{u}\|^{2}+\frac{\lambda^{2}}{4}\|\nabla \Delta \rho\|^{2}$.

Case 1: Using Gronwall's Lemma in (46) jointly with (45) for $\gamma=0$, we obtain $\varphi_{2}(t) \leq C$ for all $t \in[0, T]$ and $\int_{0}^{T}\left(\chi_{2}(t)+\psi_{2}(t)\right) d t \leq C$, hence (39) and (40) hold. 
Case 2: We obtain from (46)

$$
\varphi_{2}^{\prime}(t) \leq C \varphi_{2}^{2}(t)+C_{1}
$$

We observe that

$$
C \varphi_{2}^{2}+C_{1} \leq 2 C \varphi_{2}^{2}
$$

for all $\varphi_{2} \geq\left(C_{1} / C\right)^{1 / 2}$. Then, either we have $0 \leq \varphi_{2}(t) \leq L$ for all $t \geq 0$ or there exist some interval $\left[t_{1}, t_{2}\right]$ with $t_{2} \geq t_{1}$ for which $\left\|\varphi_{2}\left(t_{1}\right)\right\|^{2}=L$ and for all $t \in\left[t_{1}, t_{2}\right]$ one has $\left\|\varphi_{2}(t)\right\|^{2} \geq L$, where we take $L=\max \left\{\left(C_{1} / C\right)^{1 / 2}, 1,\left\|\varphi_{2}(0)\right\|\right\}$. Then, if we consider the second case, thanks to the choice of $L$, the differential inequality $\frac{d}{d t} \varphi_{2} \leq C \varphi_{2}^{2}$ holds in the interval $\left[t_{1}, t_{2}\right]$, or equivalently,

$$
\frac{d}{d t} \ln \varphi \leq C \varphi \quad \text { in }\left[t_{1}, t_{2}\right]
$$

Then, multiplying this differential inequality by $e^{\bar{\gamma} t}$ this gives

$$
\frac{d}{d t}\left(e^{\bar{\gamma} t} \ln \varphi_{2}\right) \leq C e^{\bar{\gamma} t} \varphi_{2}+\bar{\gamma} e^{\bar{\gamma} t} \ln \varphi_{2} \quad \text { in }\left[t_{1}, t_{2}\right] .
$$

Taking into account the inequality $\ln \varphi_{2} \leq k+k \varphi_{2}$, for all $\varphi_{2}>0$ for a certain $k>0$ and integrating (47) between $t$ and $t_{1}$ for any time $t \in\left[t_{1}, t_{2}\right]$, one gets

$$
e^{\bar{\gamma} t} \ln \vartheta(t)-e^{\bar{\gamma} t_{1}} \ln \vartheta\left(t_{1}\right) \leq(C+\bar{\gamma} k) \int_{t_{1}}^{t} e^{\bar{\gamma} \tau} \vartheta(\tau) d \tau+\bar{\gamma} k \int_{t_{1}}^{t} e^{\bar{\gamma} \tau} d \tau
$$

hence, thanks to (45),

$$
\begin{aligned}
\ln \vartheta(t)-e^{-\gamma\left(t-t_{1}\right)} \ln L & \leq(C+\gamma k) e^{-\gamma t} \int_{t_{0}}^{t} e^{\gamma \tau} \vartheta(\tau) d \tau+\gamma k e^{-\gamma t} \int_{t_{0}}^{t} e^{\gamma \tau} d \tau \\
& \leq C(C+\gamma k):=D
\end{aligned}
$$

Since $e^{-\gamma\left(t-t_{1}\right)} \ln L \leq \ln L$ (here, $L \geq 1$ is used), we have that $\ln \frac{\varphi_{2}(t)}{L} \leq D$, which implies,

$$
\varphi_{2}(t) \leq L e^{D}, \quad \forall t \in\left[t_{1}, t_{2}\right]
$$


Consequently $\varphi_{2}(t) \leq L e^{D}$, for all $t \geq 0$, hence one has (41).

Multiplying by $e^{\gamma t}$ in (46) and considering (41) we get the estimate (42).

Remark 7 In the $2 D$ case is possible to obtain the same regularity results given in Corollary 5 but without smallness constraints on the data.

\section{Global existence as the external force decay exponentially}

Theorem 8 Let $\Omega \subset \mathbb{R}^{3}$. Assume $\boldsymbol{u}_{0} \in \boldsymbol{V}, \rho_{0} \in H_{N}^{2}(\Omega)$ verifying (2) and $\sup _{t \geq 0} e^{\gamma t}\|\boldsymbol{f}\|_{L^{2}(\Omega)}^{2}<\infty$. If $\left\|\nabla \boldsymbol{u}_{0}\right\|,\left\|\Delta \rho_{0}\right\|$ and $\left\|e^{\gamma t / 2} \boldsymbol{f}\right\|_{L^{\infty}\left(0, \infty ; L^{2}(\Omega)\right)}$ are small enough, then there exists a unique strong solution $(\boldsymbol{u}, \rho)$ of Problem (1) in $(0, \infty)$ and a positive constant $\gamma^{*} \leq \gamma$ such that

$$
\begin{gathered}
e^{\gamma^{*} t}\|(\boldsymbol{u}(t), \rho(t))\|_{H^{1} \times H^{2}} \leq C, \quad \forall t \geq 0, \\
\int_{0}^{t} e^{\theta s}\left\|\left(\boldsymbol{u}(s), \rho(s), \boldsymbol{u}_{t}(s), \rho_{t}(s)\right)\right\|_{H^{2} \times H^{3} \times L^{2} \times H^{1}}^{2} d s \leq C, \quad \forall t \geq 0 .
\end{gathered}
$$

for any $\theta: 0 \leq \theta \leq \gamma^{*}$ and $\theta<\gamma$ (indeed, if $\gamma^{*}<\gamma$ then $\theta=\gamma^{*}$ is valid).

Proof: Multiplying (20) by $e^{\gamma^{*} t}$ with $\gamma^{*}>0$ (to be chosen), we have

$$
\left(e^{\gamma^{*} t} \varphi_{2}(t)\right)^{\prime}+e^{\gamma^{*} t}\left(\chi_{2}(t)+\psi_{2}(t)\right) \leq C e^{\gamma^{*} t} \varphi_{2}(t)^{3}+C e^{\gamma^{*} t}\|\boldsymbol{f}\|^{2}+\gamma^{*} e^{\gamma^{*} t} \varphi_{2}(t) .
$$

Now, by choosing adequate $\gamma^{*}$ such that $\gamma^{*} \varphi_{2}(t)<\psi_{2}(t)$, we get

$$
\widetilde{\varphi}_{2}(t)^{\prime}+\widetilde{\chi}_{2}(t)+\widetilde{\psi}_{2}(t) \leq C e^{-2 \gamma^{*} t} \widetilde{\varphi}_{2}(t)^{3}+C e^{\gamma^{*} t}\|\boldsymbol{f}\|^{2}
$$

being $\widetilde{\varphi}_{2}(t)=e^{\gamma^{*} t} \varphi_{2}(t), \widetilde{\chi}_{2}(t)=e^{\gamma^{*} t} \chi_{2}(t)$ and $\widetilde{\psi}_{2}(t)=C_{1} e^{\gamma^{*} t} \psi_{2}(t)$ where $C_{1}>0$ a constant depending on the data. Proceeding with similar arguments as in Theorem 4 we prove (48) whenever $\left\|\nabla \boldsymbol{u}_{0}\right\|,\left\|\Delta \rho_{0}\right\|$ and $\sup _{t \geq 0} e^{\gamma t}\|\boldsymbol{f}\|_{L^{2}(\Omega)}$ 
are small enough. Then, multiplying (20) by $e^{\theta t}$ and integrating in $(0, t)$, we can arrive at (49).

Corollary 9 Assume hypotheses of Theorem 8, $\boldsymbol{u}_{0} \in \boldsymbol{V} \cap \boldsymbol{H}^{2}(\Omega), \rho_{0} \in H_{N}^{3}(\Omega)$, $\sup _{t>0}\|\boldsymbol{f}\|_{H^{1}(\Omega)}<\infty$ and $\sup _{t>0} e^{\gamma t}\left\|\boldsymbol{f}_{t}\right\|_{L^{6 / 5}(\Omega)}^{2}<\infty(\gamma$ is given in Theorem 8). Then, the unique strong solution $(\boldsymbol{u}, \rho)$ of Problem (1) in $(0, \infty)$ given in Theorem 8 verifies

$$
\begin{gathered}
\left\|e^{\theta t}\left(\boldsymbol{u}(t), \rho(t), \boldsymbol{u}_{t}(t), \rho_{t}(t)\right)\right\|_{H^{2} \times H^{3} \times L^{2} \times H^{1}} \leq C, \quad \forall t \geq 0, \\
\int_{0}^{t} e^{\theta s}\left\|\left(\boldsymbol{u}(s), \rho(s), \boldsymbol{u}_{t}(s), \rho_{t}(s)\right)\right\|_{H^{3} \times H^{4} \times H^{1} \times H^{2}}^{2} \leq C .
\end{gathered}
$$

for any $\theta$ as in Theorem 8. Moreover, if $\rho_{0} \in H_{N}^{4}(\Omega)$

$$
\begin{aligned}
& e^{\theta t}\left\|\left(\sigma^{1 / 2}(t) \boldsymbol{u}(t), \rho(t), \sigma^{1 / 2} \boldsymbol{u}_{t}(t), \rho_{t}(t)\right)\right\|_{H^{3} \times H^{4} \times H^{1} \times H^{2}} \leq C, \\
& \forall t \geq 0, \\
& \int_{0}^{t} e^{\theta s}\left\|\left(\sigma^{1 / 2} \boldsymbol{u}, \rho, \sigma^{1 / 2} \boldsymbol{u}_{t}, \rho_{t}, \sigma^{1 / 2} \boldsymbol{u}_{t t}, \rho_{t t}\right)\right\|_{H^{4} \times H^{5} \times H^{2} \times H^{3} \times L^{2} \times H^{1}}^{2} \\
& \leq C, \quad \forall t \geq 0 .
\end{aligned}
$$

Proof: Multiplying (30) by $e^{\theta t}$ and taking into account (49) and $\int_{0}^{t} e^{\theta \tau}\left\|\boldsymbol{f}_{t}\right\|_{L^{6 / 5}}^{2} \leq$ $C$, we get (50) and (51) for $\left(\boldsymbol{u}_{t}, \rho_{t}\right)$. Now, multiplying (33), (34) and (35) by $e^{\theta t}$, it is easy to deduce (50) and (51) for $(\boldsymbol{u}, \rho)$. The rest of estimates can be obtained as in the proof of Theorem 4.

Remark 10 The above result also is valid in the $2 D$ case without constraints on the data.

Remark 11 By using the results of the above sections together with the arguments used by Heywwod [8], it is possible to prove, when $\Gamma$ is of class $C^{\infty}$, the 
following regularity for the solution

$$
\boldsymbol{u} \in C^{\infty}((0, \infty) \times \bar{\Omega}), \quad \rho \in C^{\infty}((0, \infty) \times \bar{\Omega})
$$

Remark 12 In this work we have used technics which are similar to those ones used in [14], in the case of 2-dimensional viscous compressible flows.

Acknowledgments. The second, third and fourth authors are partially supported by DGI-MEC (Spain), Grant BFM2003-06446 and CGCI MECD-DGU Brazil/Spain Grant 117/06. The authors gratefully acknowledge the referee who have pointed the paper [14].

\section{References}

[1] Antoncev, S. N.; Kazhikov, A. V.; Monakhov, V. N., Boundary value problems in mechanics of nonhomogeneous fluids, North-Holland, 1990.

[2] Beirão da Veiga, H., Diffusion on viscous fluids, existence and asymptotic properties of solutions, Ann. Sc. Norm. Sup. Pisa, 10, (1983), 341-355.

[3] Beirão da Veiga, H., Long time behaviour of the solutions to the NavierStokes equations with diffusion, Nonl. Anal.- Theory, Meth. and Applic., vol. 27, no. 11, (1996), 1229-1239.

[4] Damázio, P. D.; Rojas-Medar, M. A., On the convergence rate of semiGalerkin approximations for the equations of viscous fluids in the presence of diffusion, Mat. Contemp, 15, (1998), 105-125.

[5] Frank, D. A.; Kamenetskii, V. I., Diffusion and Heat Transfer in Chemical Kinetics, Plenum Press, (1969). 
[6] Friedman, A., Partial Differential Equations, Holt, Rinehart and Winston, New York, (1976).

[7] Guillén-González, F., Sobre un modelo asintótico de difusión de masa para fluidos incompresibles, viscoso y no homogéneos, Proceedings of the Third Catalan Days On Applied Mathematics (1996) 103-114, ISBN: 84-87029-876.

[8] Heywood, V. I., An error estimate uniform in time for spectral Galerkin approximations of the Navier-Stokes problem, Pacific J. Math, 98, (1982), 333-345.

[9] Guillén-González, F.; Damázio, P.; Rojas-Medar, M. A., Approximation by an iterative method for regular solutions for incompressible fluids with mass diffusion, J. Math. Anal. Appl., 326, no. 1, (2007), 468-487.

[10] Heywood, J.G.; Rannacher, R., Finite element aproximation of the nonstationary Navier-Stokes problem, I. Regularity of solutions and second order error estimates for spacial discretization, SIAM J. Num. Anal., 19, 2 (1982), $275-311$.

[11] Heywood, J.G., The Navier-Stokes equations: on the existence, regularity and decay of solutions, Indiana Univ. Math. J. 29, no. 5, (1980), 639-681.

[12] Kazhikov, A. V.; Smagulov, Sh., The correctness of boundary-value problems in a diffusion model of inhomogeneous fluid, Dokl. Akad. Nauk. SSSR, 234 (1977), 330-332.

[13] Ladyzhenkaya, O. A., The Mathematical Theory of Viscous Incompressible Fluid, Gordon and Breach, New York, (1969). 
[14] Padula, M., Existence og global solutions for 2-dimensional viscous compressible flows, J. Functional Analysis, 69 (1986), 1-20.

[15] Salvi, R., On the existence of weak solutions of boundary-value problems in a diffusion model of an inhomogeneous liquid in regions with moving boundaries, Portugaliae Math. 43 (1986), 213-233.

[16] Simon, J., Non-Homogeneous Viscous Incompressible Fluids: Existence of Velocity, Density and Pressure, SIAM J. Math. Anal. 21, no. 5, (1990), 1093-1117.

[17] Secchi, P., On the motion of viscous fluids in the presence of diffusion, SIAM J. Math. Anal. 19 (1988), 22-31.

[18] Secchi, P., On the inicial value problem for the equations of motion of viscous incompressible fluids in the presence of diffusion, Bollettino U.M.I., 6 1-B, 1982, 1117-1130.

[19] Temam, R., Navier-Stokes equations, theory and numerical analysis, North-Holland, Amsterdam, 1979.

Departamento de Matemática UFPR, Centro Politécnico Caixa Postal 019081 Jd. das Américas 81531-990, Curitiba, PR, Brazil E-mail: pedro@mat.ufpr.br

Universidad del Bío-Bío

Facultad de Ciencias Departamento de Ciencias Básicas Campus Fernando May Casilla 447, Chillán, Chile E-mail: marko@ueubiobio.cl
Depto. E.D.A.N.

University of Sevilla

Aptdo. 1160, 41080 Sevilla

Spain

E-mails: guillen@us.es juanvi@us.es 